$$
\text { Pontifícia Universidade C Católica }
$$

Júlio César Pereira de Oliveira

\begin{abstract}
A Relevância da Colaboração entre Empresa e suas Contratadas para o Desempenho Operacional: Um Estudo de Caso
\end{abstract}

Dissertação de Mestrado

Dissertação apresentada como requisito parcial para obtenção do grau de Mestre pelo Programa de PósGraduação em Engenharia de Produção do Departamento de Engenharia Industrial da PUCC-Rio.

Orientador: Prof. Leonardo Junqueira Lustosa

Rio de Janeiro

Março de 2009 


$$
\text { Pontifícia } \text { Universidade }_{\text {Do Rio de Janeiro }}
$$

Júlio César Pereira de Oliveira

\title{
A Relevância da Colaboração entre Empresa e suas Contratadas para o Desempenho Operacional: Um Estudo de Caso
}

\begin{abstract}
Dissertação apresentada como requisito parcial para obtenção do título de Mestre pelo Programa de PósGraduação em Engenharia de Produção da PUC-Rio. Aprovada pela Comissão Examinadora abaixo assinada.
\end{abstract}

Prof. Leonardo Junqueira Lustosa

Orientador

Departamento de Engenharia Industrial - PUC-Rio

Prof. José Geraldo Vidal Vieira Departamento de Engenharia de Produção UFSCAR - Campus Sorocaba

Profa. Maria Ângela Campelo de Melo Departamento de Administração - PUC - Rio

Prof. José Eugenio Leal Coordenador Setorial do Centro Técnico Científico - PUC-Rio 
Todos os direitos reservados. É proibida a reprodução total ou parcial do trabalho sem autorização da universidade, do autor e do orientador.

\section{Júlio César Pereira de Oliveira}

Graduou-se em Engenharia de Produção pela Universidade do Estado do Pará em 2006. Foi estagiário nas áreas de PCP e logística. Em 2005 atuou como consultor de PCP no Arranjo Produtivo Local (APL) São Miguel do Guamá no Estado do Pará, onde fez consultoria para empresas cerâmicas. Atualmente é engenheiro de uma empresa de transporte ferroviário.

Ficha Catalográfica

Oliveira, Júlio César Pereira de

A relevância da colaboração entre empresa e suas contratadas para o desempenho operacional : um estudo de caso / Júlio César Pereira de Oliveira ; orientador: Leonardo Junqueira Lustosa. - 2009.

93f. : il. ; $30 \mathrm{~cm}$

Dissertação (Mestrado em Engenharia Industrial) - Pontifícia Universidade Católica do Rio de Janeiro, Rio de Janeiro, 2009.

Inclui bibliografia

1. Engenharia industrial - Teses. 2. Terceirização. 3. Colaboração. 4. Relacionamento colaborativo. 5. Desempenho operacional. I. Lustosa, Leonardo Junqueira. II. Pontifícia Universidade Católica do Rio de Janeiro. Departamento de Engenharia Industrial. III. Título.

CDD: 658.5 
À minha mãe Célia, ao meu pai Mário e irmãos Giselle e Mário Jr., pelos incentivos e demonstrações de carinho e amor. 


\section{Agradecimentos}

Agradeço a Deus e a Virgem de Nazaré por mais essa oportunidade.

A Capes, pelo apoio financeiro recebido durante o curso.

Ao Departamento de Engenharia Industrial, pelo apoio e excelente infra-estrutura.

Ao meu orientador Leonardo Junqueira Lustosa, pelos ensinamentos, estímulos e confiança.

Aos funcionários da empresa foco de minha pesquisa, Daniel Schaefer, Ermeci, André Abdalla os quais foram de real importância para a realização dessa pesquisa.

A minha família, pelo apoio incondicional de sempre.

A todos os amigos que conquistei no mestrado, pelo apoio, incentivo, dicas e horas dispensadas de estudo. 


\section{Resumo}

Oliveira, Júlio César Pereira de; Lustosa, Leonardo Junqueira (Orientador). A relevância da colaboração entre empresa e suas contratadas para o desempenho operacional: Um estudo de caso. Rio de Janeiro, 2009. 93p. Dissertação de Mestrado - Departamento de Engenharia Industrial, Pontifícia Universidade Católica do Rio de Janeiro.

A terceirização na manufatura e em serviços tem proporcionado às empresas vantagens estratégicas como menor custo devido a economias de escala (no fornecedor), maior competência técnica, rápida aquisição de novas competências, menor necessidade de investimento, maior foco em produtos e mercados entre outras. Nesse cenário, ganha importância o relacionamento colaborativo em que as empresas mudam a ênfase do benefício imediato em transações isoladas para estratégias que objetivam transações repetitivas com parceiras preferenciais em busca de eficiência e eficácia no longo prazo. Através de um estudo de caso, a presente pesquisa busca averiguar, na relação de uma empresa com algumas de suas importantes parceiras, até que ponto a eficiência das operações está associada aos elementos-chave da colaboração encontrados na literatura. A pesquisa foi realizada numa companhia telefônica durante um período de três meses, no qual, um questionário estruturado foi aplicado ao pessoal de gerência e supervisão. Os dados da pesquisa foram confrontados com indicadores de desempenho compilados periodicamente pela companhia para medir e monitorar a atuação das terceiras. Os resultados sugerem que há, de fato, uma correlação positiva entre os níveis dos fatores relacionados a práticas colaborativas mais significativas e o bom desempenho operacional da parceria.

\section{Palavras-chave}

Terceirização, Colaboração, Relacionamento Colaborativo, Desempenho Operacional. 


\section{Abstract}

Oliveira, Júlio César Pereira de; Lustosa, Leonardo Junqueira (Advisor). The relevance of collaboration between a company and its third parties for operational performance: A case study. Rio de Janeiro, 2009. 93p. MSc Dissertation - Departamento de Engenharia Industrial, Pontifícia Universidade Católica do Rio de Janeiro.

Outsourcing in manufacturing as well as in services has granted enterprises strategic advantages such as lower cost yielded by economies of scale (at the vendor), greater technical competence, fast acquisition of new competencies, less investment needs, and sharper focus on products and markets, among others. This scenario promotes the collaborative relationship whereby enterprises change their emphasis from the immediate gain in individual transactions to strategies that seek repetitive transactions with preferential partners for enhancing efficiency and effectiveness in the long run. By means of a case study this research aims to verify, in the relationships of an enterprise and some of its important suppliers, to what degree the efficiency of operations is associated to the key elements of collaboration found in the literature. The research has been conducted in a telephone company along a three-month period when a structured questionnaire was filled out by the managerial and supervisory personnel. The data from the survey were confronted with the performance indicators regularly collected by the company for measuring and monitoring the outsourced services. The results suggest that there is, indeed, a positive correlation between the levels of the more important factors related to the collaborative practices and the good performance of the partnership.

\section{Keywords}

Outsourcing, Collaboration, Collaborative Relationship, Operational Performance. 


\section{Sumário}

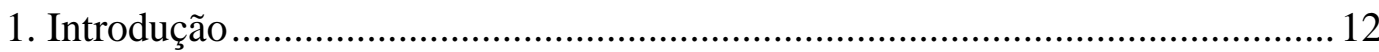

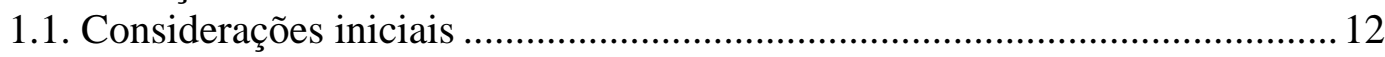

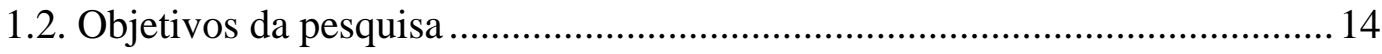

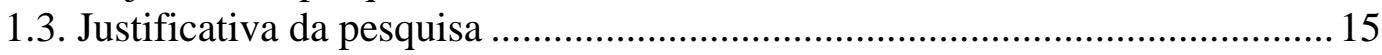

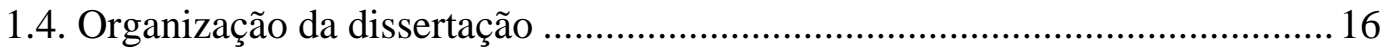

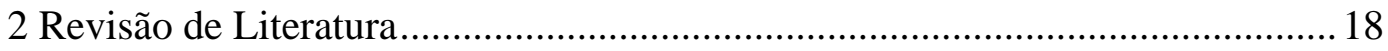

2.1. As novas tendências de reestruturação industrial ........................................... 18

2.1.1. O Conceito de terceirização, suas vantagens e implicações ......................... 20

2.2. Benefícios, barreiras, conceitos e fatores da colaboração .............................. 22

2.2.1. Benefícios da colaboração e barreiras na implantação do comportamento

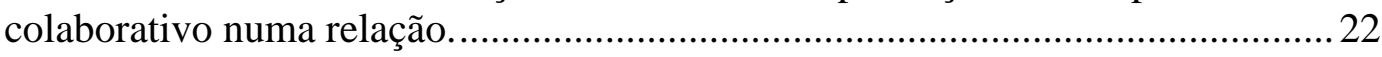

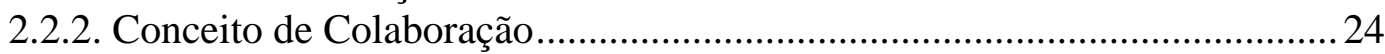

2.2.3. Fatores que influenciam no relacionamento colaborativo............................ 28

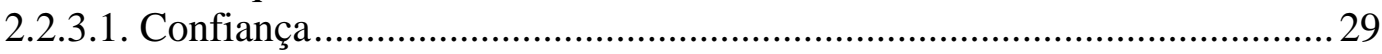

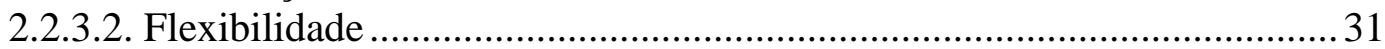

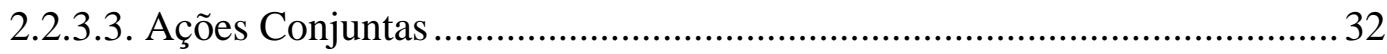

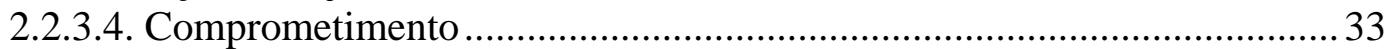

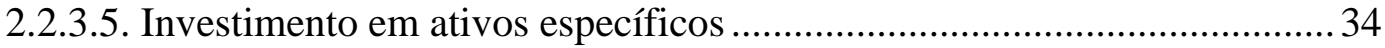

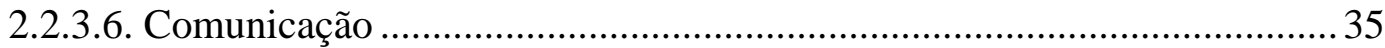

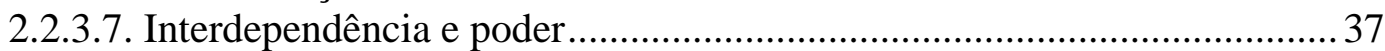

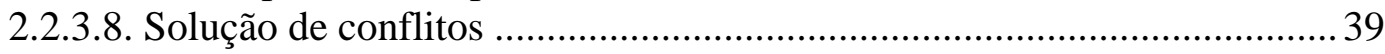

2.3. O papel da colaboração na melhoria de desempenho.................................... 40

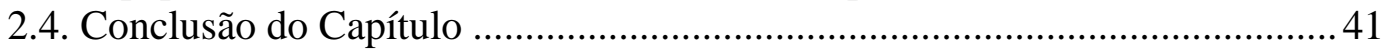

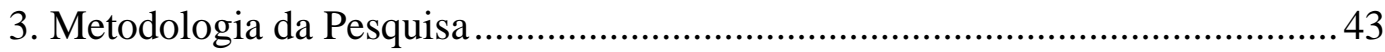

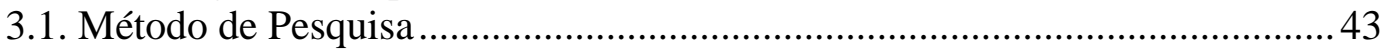

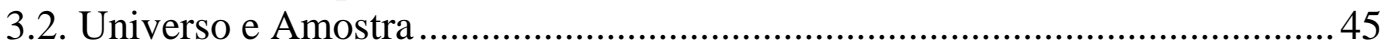

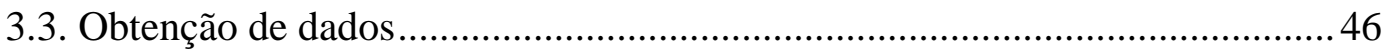

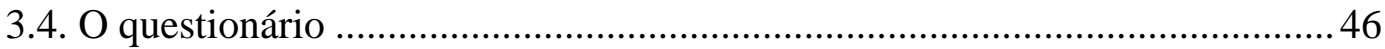

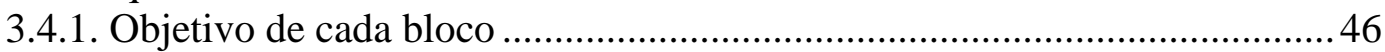

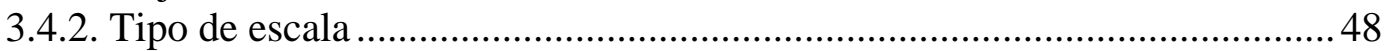

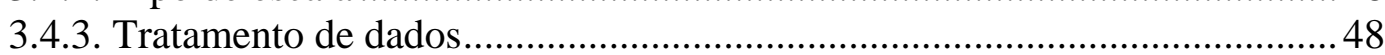

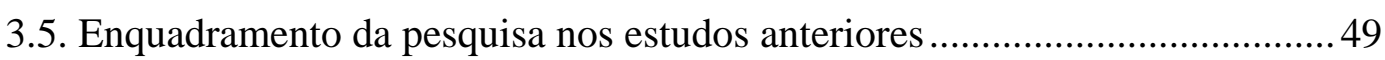

3.6. Principais pontos fortes e pontos fracos da metodologia da pesquisa............ 50

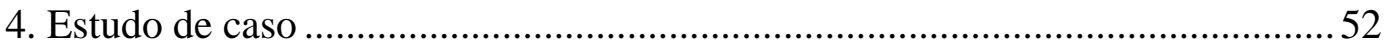

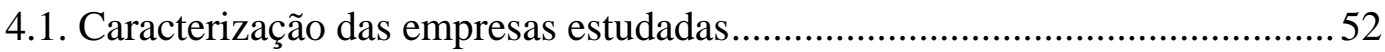

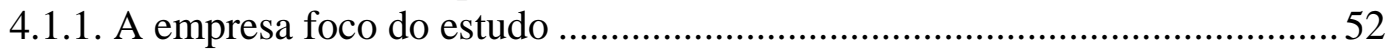

4.1.2. Sistema de Gestão da Qualidade (SGQ) - Avaliação e qualificação de

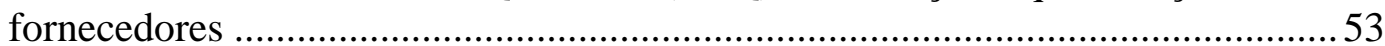

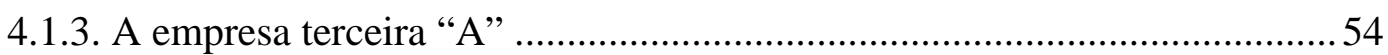

4.1.3.1. Indicadores de desempenho operacional da empresa terceira " $A$ "............55 55

4.1.4. A empresa terceira "B”.............................................................................. 56

4.1.4.1. Indicadores de desempenho operacional da empresa terceira “B”............ 57

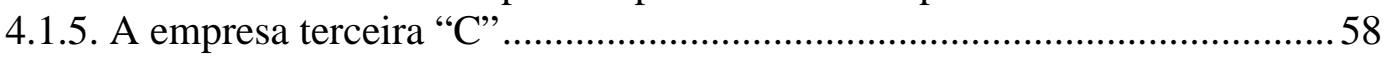

4.1.5.1. Indicadores de desempenho operacional da empresa terceira “C”........... 58 
5. Análise e discussão dos resultados

60

5.1. Comparação entre opiniões obtidas sobre a colaboração em nível de gerência média e supervisão de pessoal

5.1.1. Resultados referentes à empresa terceira “A” em nível de gerência e supervisão

5.1.2. Resultados referentes à empresa terceira “B” em nível de gerência e supervisão

5.1.3. Resultados referentes à empresa terceira “C” em nível de gerência e supervisão

5.2. A Relevância do alto nível de colaboração sobre o desempenho operacional na relação entre a Companhia e suas terceiras.

5.3. Questões que podem vir a inibir o relacionamento entre a Companhia e suas terceiras

5.4. Implicações gerenciais para melhorar e/ou aumentar os níveis de colaboração

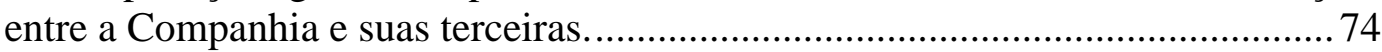

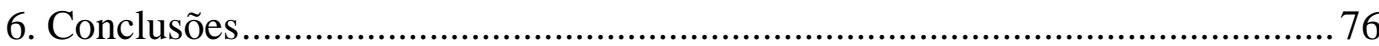

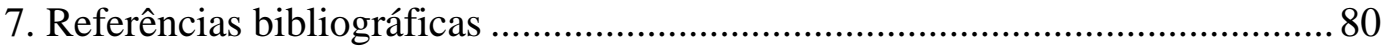

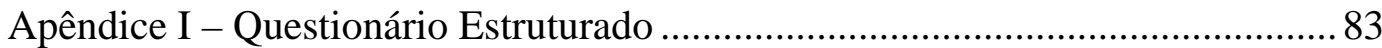

Apêndice II - Indicadores de desempenho da empresa “A” .................................. 88

Apêndice III - Indicadores de desempenho da empresa "B” "................................. 90

Apêndice IV - Indicadores de desempenho da empresa "C”".................................91 


\section{Lista de Figuras}

Figura 2.1 - O relacionamento colaborativo e seus benefícios relacionados a

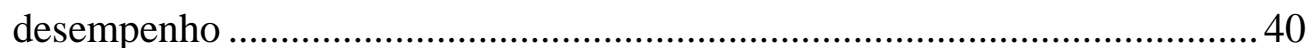

Gráfico 5.1 - Comparação entre os fatores obtidos pelas empresas terceiras........ 67

Gráfico 5.2 - Níveis de relacionamento colaborativo das empresas terceiras. ...... 68

Gráfico 5.3 - Ranking IQF por PSR todos os segmentos - Comparação entre os anos de 2006 e 2007 das empresas terceiras................................................ 71 


\section{Lista de Tabelas}

Quadro 2.1 - Vantagens da colaboração nos níveis financeiro, operacional e

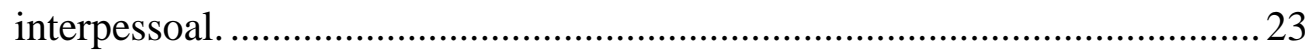

Quadro 2.2 - Definição de colaboração segundo alguns autores ........................... 26

Quadro 2.3 - Definição do fator confiança segundo alguns autores ..................... 30

Quadro 2.4 - Definição do fator flexibilidade segundo alguns autores ................. 31

Quadro 2.5 - Definição do fator ação conjunta segundo alguns autores .............. 32

Quadro 2.6 - Definição do fator comprometimento segundo alguns autores ....... 33

Quadro 2.7 - Definição do fator investimento em ativos específicos segundo

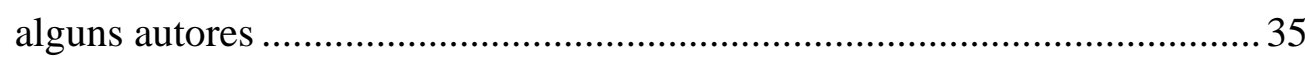

Quadro 2.8 - Definição do fator comunicação segundo alguns autores ............... 37

Quadro 2.9 - Definição dos fatores interdependência e poder segundo alguns

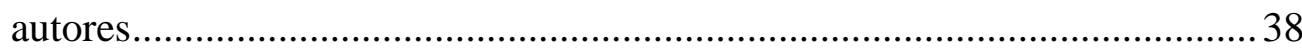

Quadro 3.1 - Pesquisas realizadas anteriormente envolvendo o tema desse estudo

Tabela 5.1 - Consolidação dos fatores intrínsecos à colaboração - Empresa terceira "A"

Tabela 5.2 - Consolidação dos fatores intrínsecos à colaboração - Empresa terceira "B".

Tabela 5.3 - Consolidação dos fatores intrínsecos à colaboração - Empresa terceira "C"

Tabela 5.4 - Ranking IQF das empresas terceiras para todos os segmentos......... 69

Tabela 5.5 - Ranking IQF das empresas terceiras por Geografia ......................... 70 